\title{
Phase locking in networks of synaptically-coupled McKean relaxation oscillators
}

\author{
S Coombes \\ Nonlinear and Complex Systems Group, Department of Mathematical Sciences, \\ Loughborough University, Loughborough, Leicestershire, LE11 3TU, UK. \\ e-mail: S.Coombes@lboro.ac.uk \\ Tel: (UK) 01509 22 3191, Fax: (UK) 01509 22 3969
}

\begin{abstract}
We use geometric dynamical systems methods to derive phase equations for networks of weakly connected McKean relaxation oscillators. We derive an explicit formula for the connection function when the oscillators are coupled with chemical synapses modeled as the convolution of some input spike train with an appropriate synaptic kernel. The theory allows the systematic investigation of the way in which a slow recovery variable can interact with synaptic time scales to produce phase-locked solutions in networks of pulse coupled neural relaxation oscillators. The theory is exact in the singular limit that the fast and slow time scales of the neural oscillator become effectively independent. By focusing on a pair of mutually coupled McKean oscillators with alpha function synaptic kernels, we clarify the role that fast and slow synapses of excitatory and inhibitory type can play in producing stable phase-locked rhythms. In particular we show that for fast excitatory synapses there is coexistence of a stable synchronous, a stable anti-synchronous, and one stable asynchronous solution. For slower synapses the anti-synchronous solution can lose stability, whilst for even slower synapses it can regain stability. The case of inhibitory synapses is similar up to a reversal of the stability of solution branches. Using a return-map analysis the case of strong pulsatile coupling is also considered. In this case it is shown that the synchronous solution can co-exist with a continuum of asynchronous states.
\end{abstract}




\section{Introduction}

The phenomenon of phase locking in networks of synaptically interacting neural oscillators is common to many areas of neuroscience. Synchronous activity in thalamic and hippocampal networks [1,2], anti-synchronous rhythms in central pattern generators [3], and synchronized cortical oscillations [4] are all examples of phase-locked behavior that depend crucially upon the intrinsic mechanisms of neural oscillation as well as the nature of synaptic coupling. Synchronous activity is especially important for synaptic plasticity as cells firing within some common window of time are capable of increasing the strength of the synapses between them. Moreover, synchronous spindle oscillations are observed during sleep or anesthesia, behavior which is itself supported by oscillations within the reticular thalamic nucleus [5]. Simulation studies have played an important role in uncovering the way in which synchronization depends upon network architectures and single neuron properties. (See for example $[6,7,8,5,9,10,11]$.) In a complementary manner mathematical tools are being used to clarify the role of synaptic time courses in generating network synchrony for analytically tractable single neuron models. The biophysical mechanisms underlying neural oscillations are typically modelled in terms of a system of differential equations describing the generation of an action potential due to the activationdeactivation of various voltage-dependent ionic gates [12]. Unfortunately, analyzing a network of such oscillators is a difficult task due to the complexity of the single-neuron model. This motivates the application of techniques such as invariant manifold theory [13] and averaging theory [14] to reduce the network dynamics to a system in which the relative phase between oscillators is the relevant dynamical variable [15, 16, 17]. For example, there has been a comprehensive study of integrate-and-fire (IF) and related spike response networks within a dynamical systems framework [18, 19, 20, 21, 22, 23, 24, 25] as well as a growing number of studies on more biophysically realistic relaxation oscillator models [26, 27, 28, 29, 30, 31, 32]. From the work on IF systems, and making extensive use of the fact that the systems behave linearly between spiking events, it is apparent that IF models of spiking cells can form coherent rhythms with purely inhibitory signals. If the time course for the onset and offset of the inhibitory signal is long enough, and sufficiently weak, inhibition alone can stabilize a synchronous rhythm. (See [25] for a discussion of strong coupling instabilities.) The treatment of more biophysically realistic cells that in- 
corporate some notion of refractoriness, for instance with the inclusion of gating variables, is much less amenable to an exact treatment. Not only are the equations for a network of high dimension, but they are inherently nonlinear for all times. Perhaps the most significant mathematical progress in the study of population rhythms for high dimensional neural systems has come about with the use of geometric singular perturbation methods. A recent review of this mathematical tool can be found in [33]. For example, this technique may be used to show that for neurons possessing a slow recovery variable an inhibitory network alone cannot exhibit stable synchronous oscillations if the synapses are fast [34]. The experimental observation of synchronous oscillations in inhibitory networks (for example [1]) would therefore suggest that there may be some interaction between recovery and synaptic time scales which, in the right circumstances, can lead to stable synchronous rhythms. Indeed, although much research has been done on how the interaction of time scales affects synchrony, novel effects continue to emerge so that a general picture is as yet incomplete [32]. By focusing on the McKean neuron model [35], a caricature of the well known FitzHugh-Nagumo neuron model, we are able to make precise statements about synchronization properties in relation to these fundamental time scales. The analysis is undertaken with a combination of geometric singular perturbation theory and the theory of coupled limit cycle oscillators, and assumes both strong relaxation and weak coupling. For fast synapses we are also able to treat the case of strong pulsatile coupling with an alternative return-map analysis.

In section 2 we introduce the McKean model and discuss its description in the strong relaxation limit using the binary terminology originally introduced by Abbott [36]. In section 3 we develop an exact treatment of networks of weakly synaptically interacting neural relaxation oscillators of the McKean type. The McKean model possesses a onedimensional variable responsible for determining the shape of an action potential as well as providing the model neuron with a natural refractoriness. The fast relaxation limit describes the case when the rise and fall time of the action potential is essentially instantaneous. Using averaging theory to construct phase equations for networks of McKean oscillators shows quite clearly, in the fast relaxation limit, that the inclusion of a recovery variable can significantly influence the synchronization properties of a network. Moreover, the phase equations are different from those of non-relaxation oscillators because they are discontinuous. A general discussion of relaxation oscillators and discontinuous phase interaction functions has recently been presented by Izhikevich [37]. The detailed discussion presented here for the McKean oscillator is entirely consistent with this formalism, with 
the added advantage of allowing an exact calculation of the phase response curve and hence an explicit form for the phase-interaction function. Somers and Kopell [38] have already shown that the synchronization of relaxation oscillators is fundamentally different from those of non-relaxation type, at least for a form of synaptic coupling called fast threshold modulation (FTM). In section 4 we analyze the case of two mutually coupled McKean oscillators in some detail. Using the theory developed in section 3 we construct the phase-locked response of the system when the synapse has a time course described by an alpha function. Consistent with previous numerical studies of the Hodgkin-Huxley system, we find that both the anti-synchronous and synchronous solution are stable for fast excitatory synapses. A similar observation also holds for relaxation oscillators possessing a cubic fast nullcline with FTM coupling [39]. Interestingly, for slower synapses the antisynchronous solution can destabilize, whilst for even slower synapses it can restabilize. A pair of stable/unstable asynchronous solutions is also found for fast synapses and may be thought of as the novel behavior to be associated with the McKean oscillator, in the limit of fast relaxation. In section 5 we relax the assumption of weak coupling and show how to treat the binary model as a type of IF neuron with a state dependent threshold. This allows a formulation of the response of a binary neuron to pulsatile stimuli and hence a study of networks of pulse coupled binary relaxation oscillators. Using a return-map analysis along similar lines to that introduced in the seminal paper of Mirollo and Strogatz [40] (for the study of pulse coupled IF neurons) we show that the system can support a synchronous solution co-existing with a continuum of asynchronous states. Finally in section 6 we summarize the results of our analysis and discuss applications and extensions of the theory.

\section{The McKean model}

The McKean model [35] may be regarded as a planar neuron model capable of generating voltage pulses. Alternatively one may regard it as a reduction of the four dimensional Hodgkin-Huxley neuron model which trades a detailed description of the usual HodgkinHuxley gating variables for a slow recovery variable and analytical tractability. Even so, a general analysis of networks of such neurons has yet to be given. However, mathematical progress can be made under the simplifying assumption of fast relaxation which allows one to use some of the tools of geometric singular perturbation theory. The equations for 


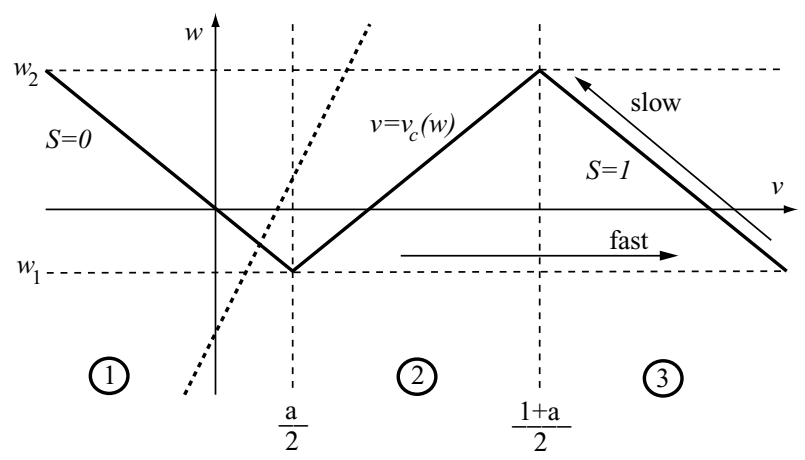

Fig. 1. The phase plane for the McKean model has a nullcline with an $N$ shape (thick solid line) corresponding to $\dot{v}=0$ and a linear one associated with $\dot{w}=0$ (thick dashed line). In this figure the stable excitable fixed point lies at the intersection of the two nullclines on the $S=0$ branch. a single two-dimensional McKean oscillator take the form

$$
\begin{aligned}
\mu \dot{v} & =f(v)-w-w_{0}+I+\epsilon X(t) \\
\dot{w} & =v-\gamma w-v_{0},
\end{aligned}
$$

where the nonlinear function $f(v)$ is given by

$$
f(v)= \begin{cases}-v, & v<a / 2 \\ v-a, & a / 2<v<(1+a) / 2 \\ 1-v, & v>(1+a) / 2\end{cases}
$$

and is a piecewise linear caricature of the cubic FitzHugh-Nagumo nonlinearity $f(v)=$ $v(1-v)(v-a)$. The variable $v$ corresponds to a membrane potential whilst $w$ is associated with the recovery property of a neuron. The parameters $a, \mu, w_{0}, v_{0}$ and $\gamma$ may be considered as combinations of membrane reversal potentials and conductance properties whilst $I$ is a constant input current. The term $\epsilon X(t)$ represents a time varying external input signal of strength $\epsilon$. In the absence of any input $(\epsilon=0)$ the system has nullclines defined by $f(v)=w+w_{0}-I$ and $w=\left(v-v_{0}\right) / \gamma$. The case when the fixed point is such that $v<a / 2$ is said to define the excitable regime. It is convenient to keep track of which branch of the nonlinear function (3) is playing a role in the dynamics. Following Abbott [36] we introduce the binary variable:

$$
S= \begin{cases}+1, & v>(1+a) / 2 \\ 0, & v<a / 2 .\end{cases}
$$


If the time-scale for the $v$ dynamics is fast compared to the time-scale for the $w$ dynamics (ie in the limit as $\mu \rightarrow 0$ ), then $v$ spends no appreciable time off of the nullclines for $\dot{v}=0$ and we may write $f(v)=S-v$. (More precisely we assume $\epsilon \ll \mu \rightarrow 0$.) Introducing $S^{+}(t)=\lim _{\delta \rightarrow 0^{+}} S(t+\delta)$ we may write the dynamics for $S(t)$ in the form

$$
S^{+}=\Theta\left(I-w_{0}+(S-a) / 2-w\right)
$$

where $\Theta(x)=1$ if $x \geq 0$ and is zero otherwise. To establish the validity of (5) we refer to figure 1 and check that $S$ switches from 0 to 1 as $w$ decreases through $w_{1}$ and that this is reversed as $w$ increases through $w_{2}$. The points $w_{1}$ and $w_{2}$ in figure 1 are easily calculated as $I-w_{0}-a / 2$ and $I-w_{0}-a / 2+1 / 2$ respectively. On the branches $S=0$ and $S=1$, the evolution of $v$ may be expressed as

$$
v=S-w-w_{0}+I
$$

This allows us to re-write the slow dynamics in the form

$$
\dot{w} \equiv G(w ; S)=-\beta w+A+S
$$

where $\beta=1+\gamma$ and $A=I-w_{0}-v_{0}$.

When $\mu=0$ the McKean model possesses an invariant manifold which may be written in the form $v=m(w)$, with

$$
m(w)= \begin{cases}m(w ; 0), & v<a / 2 \\ v_{c}(w), & a / 2<v<(1+a) / 2 \\ m(w ; 1), & v>(1+a) / 2\end{cases}
$$

where $m(w ; S)=S-w-w_{0}+I$ and $v_{c}(w)=w+a+w_{0}-I$. Note that for $\mu=0$ the two outer branches defined by $S=0$ and $S=1$ are attracting whilst the inner branch defined by $v=v_{c}(w)$ is repelling. We shall refer to the model obtained by taking the singular limit $\mu=0$ of the McKean model as the binary model. A study of a single binary neuron experiencing repetitive pulsatile stimulation can be found in [41].

\section{Phase equations}

In the oscillatory regime it is a simple matter to calculate the periodic trajectory of the system in the singular limit $\mu=0$. In this case the period of oscillation is given by 
$T=T_{1}+T_{2}$ where

$$
\begin{aligned}
& T_{1}=\int_{w_{2}}^{w_{1}} \frac{1}{G(w ; 0)} \mathrm{d} w=\frac{1}{\beta} \ln \left[\frac{\left(-\beta w_{2}+A\right)}{\left(-\beta w_{1}+A\right)}\right] \\
& T_{2}=\int_{w_{1}}^{w_{2}} \frac{1}{G(w ; 1)} \mathrm{d} w=\frac{1}{\beta} \ln \left[\frac{\left(-\beta w_{1}+A+1\right)}{\left(-\beta w_{2}+A+1\right)}\right] .
\end{aligned}
$$

In the absence of synaptic input $(\epsilon=0)$ the binary neuron $(\mu=0)$ evolves on the invariant manifolds described by $S=0$ and $S=1$. In the oscillatory regime the periodic trajectory of the binary model takes the simple form

$$
\begin{aligned}
& w(t)= \begin{cases}w_{2} \exp (-\beta t)+\frac{A}{\beta}[1-\exp (-\beta t)], & t \in\left[0, T_{1}\right) \\
w_{1} \exp \left(-\beta\left(t-T_{1}\right)\right)+\frac{A+1}{\beta}\left[1-\exp \left(-\beta\left(t-T_{1}\right)\right)\right], & t \in\left(T_{1}, T\right),\end{cases} \\
& v(t)=S-w(t)-w_{0}+I
\end{aligned}
$$

with $v(t+T)=v(t)$ and $w(t+T)=w(t)$. This trajectory (with $\epsilon=0$ ) may be parameterized by a phase variable $\theta \in[0,1)$ such that $\theta(t)=\Omega t$ with $\Omega=T^{-1}$. Hence, for some periodic functions $\Lambda_{v}$ and $\Lambda_{w}$, of period one, we may write

$$
\begin{aligned}
& w(t)=\Lambda_{w}(\theta(t)), \\
& v(t)=\Lambda_{v}(\theta(t))=-\Lambda_{w}(\theta(t))+S-w_{0}+I,
\end{aligned}
$$

so that

$$
\begin{aligned}
& \dot{w}=\Omega \frac{\mathrm{d} \Lambda_{w}}{\mathrm{~d} \theta}=G(\theta ; S), \\
& \dot{v}=\Omega \frac{\mathrm{d} \Lambda_{v}}{\mathrm{~d} \theta}=-G(\theta ; S)+\frac{\mathrm{d} S}{\mathrm{~d} \theta} .
\end{aligned}
$$

Whenever $S$ changes value there is an instantaneous jump of the system between its slow manifolds. Thus, a limit cycle attractor of the algebraic-differential system given by (14) and (15) is discontinuous. Assuming the persistence of the periodic orbit in the presence of a non-zero coupling term $(\epsilon \neq 0)$ we look for a new coordinate system that describes both the phase on the limit cycle $(\theta \in[0,1))$ and a normal coordinate $(b \in \mathbb{R})$ in its neighbourhood (that vanishes on the limit cycle). Such a coordinate transformation has been found by Ermentrout and Kopell $[42,43]$ and used to great effect in dealing with systems of coupled neural oscillators. Following closely the appendix of [42] we write the 
change of coordinates $(v, w) \rightarrow(\theta, b)$ in the form

$$
\left[\begin{array}{l}
v \\
w
\end{array}\right]=\left[\begin{array}{l}
\Lambda_{v}(\theta) \\
\Lambda_{w}(\theta)
\end{array}\right]+\left[\begin{array}{l}
M_{v}(\theta) \\
M_{w}(\theta)
\end{array}\right] b+\left[\begin{array}{l}
1 \\
1
\end{array}\right] O\left(b^{2}\right)
$$

subject to the constraints $\Lambda_{v}^{\prime} M_{v}+\Lambda_{w}^{\prime} M_{w}=0$ and $M_{v}^{2}+M_{w}^{2}=1$, where ${ }^{\prime} \equiv \mathrm{d} / \mathrm{d} \theta$. Evaluating the Jacobian of the transformation and evaluating its inverse for small $b$ shows that

$$
\left[\begin{array}{cc}
\frac{\partial \theta}{\partial v} & \frac{\partial b}{\partial v} \\
\frac{\partial \theta}{\partial w} & \frac{\partial b}{\partial w}
\end{array}\right]=\frac{1}{\rho}\left[\begin{array}{cc}
\Lambda_{v}^{\prime} & \rho M_{v} \\
\Lambda_{w}^{\prime} & \rho M_{w}
\end{array}\right], \quad \rho(\theta)=\left(\Lambda_{v}^{\prime}(\theta)\right)^{2}+\left(\Lambda_{w}^{\prime}(\theta)\right)^{2} .
$$

From the chain rule and using the above results it is easily checked that $\dot{\theta}=\Omega$. For nonzero $\epsilon$ the evolution of $v$ is modified to $v=S-w-w_{0}+I+\epsilon X(t)$ so that $\dot{w} \rightarrow \dot{w}+\epsilon X(t)$, which in turn means that $\dot{v} \rightarrow \dot{v}-\epsilon X(t)$. In this case

$$
\frac{\mathrm{d} \theta}{\mathrm{d} t}=\Omega+\epsilon R(\theta) X(t)
$$

where the response function $R(\theta)$ is given by

$$
\begin{aligned}
R(\theta) & =\frac{1}{\rho(\theta)} \frac{\mathrm{d}}{\mathrm{d} \theta}\left[\Lambda_{w}(\theta)-\Lambda_{v}(\theta)\right] \\
& =\Omega\left[\frac{1}{G(\theta ; S)}+\kappa(0) \delta(\theta)+\kappa\left(\theta_{T}\right) \delta\left(\theta-\theta_{T}\right)\right],
\end{aligned}
$$

and we use the notation $G(\theta ; S)=G\left(\Lambda_{w}(\theta) ; S\right)$ and $\theta_{T}=T_{1} / T$. The last two terms in (20) represent the contributions of $\mathrm{d} S / \mathrm{d} \theta$ to $\mathrm{d} \Lambda_{v} / \mathrm{d} \theta$. The constants $\kappa(0)$ and $\kappa\left(\theta_{T}\right)$ may be calculated by demanding that $\theta(t)$ evolve smoothly even when $S$ changes from 0 to 1 and vice-versa. This is guaranteed upon choosing

$$
\begin{aligned}
\kappa(0) & =\left(\frac{1}{G(0 ; 0)}-\frac{1}{G(1 ; 1)}\right) \\
\kappa\left(\theta_{T}\right) & =\left(\frac{1}{G\left(\theta_{T} ; 1\right)}-\frac{1}{G\left(\theta_{T} ; 0\right)}\right) .
\end{aligned}
$$

From the periodic solution given by $(11), \Lambda_{w}=w(\theta T)$ so that

$$
G(\theta ; S)= \begin{cases}\mathrm{e}^{-\beta \theta T}\left[A-\beta w_{2}\right], & S=0 \\ \mathrm{e}^{-\beta T\left(\theta-\theta_{T}\right)}\left[A+1-\beta w_{1}\right], & S=1 .\end{cases}
$$




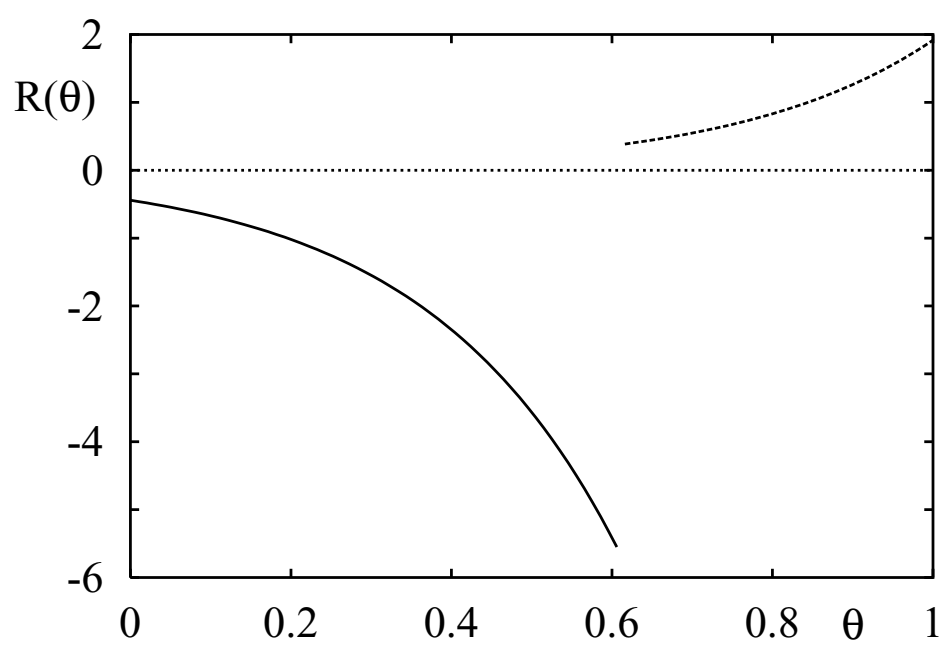

Fig. 2. Phase response curve of the binary model for $\theta \neq 0, \theta_{T}$. System parameters are taken as $v_{0}=w_{0}=0, \gamma=0.5, a=0.25$ and $I=0.5$.

Note that $S=0$ if $\theta \in\left[0, \theta_{T}\right)$ and $S=1$ if $\theta \in\left(\theta_{T}, 1\right)$. A plot of the response function (for $\theta \neq 0, \theta_{T}$ ) is shown in figure 2. We may now use the averaging theorem to construct the phase interaction function for a network of synaptically interacting binary oscillators.

\subsection{Networks, weak coupling and averaging}

By generalizing the above approach it is straightforward to see that a network of binary relaxation oscillators may be described by the system

$$
\dot{\theta}_{n}=\Omega+\epsilon \sum_{m} W_{n m} R\left(\theta_{n}\right) X_{m}(t)
$$

where $\epsilon W_{n m}$ is the strength of the coupling between oscillators $m$ and $n$. In neural systems the functions $X_{m}(t)$ represent synaptic interactions which we shall consider to be solely determined by the arrival times of pre-synaptic action potentials. Furthermore, we assume that synaptic input can be modeled as a convolution of an incoming spike train with some synaptic response kernel. Hence, we set

$$
X_{m}(t)=\int_{0}^{\infty} \eta(\tau) \sum_{j} \delta\left(\tau-t+T_{m}^{j}\right) \mathrm{d} \tau=\sum_{j} \eta\left(t-T_{m}^{j}\right)
$$

where the times $T_{m}^{j}$ represent the arrival time of the $j$ th spike from oscillator $m$. For the McKean oscillator we take the firing time to be on the upstroke of the action potential, ie the time at which the jump from the $S=0$ branch to the $S=1$ branch occurs. 
Appropriate choices of the kernel function $\eta(t)$ may be used to mimic realistic neuronal responses to spike train input. For a further discussion of the forms of delay kernel typically found in neural systems see [44]. Of course, synaptic currents may also be described using a more dynamical approach as discussed by Rubin and Terman [30] or with the FTM type of coupling studied intensively by Somers and Kopell [38, 27, 39]. For the latter type of coupling interactions are assumed to instantaneously modify the slow manifolds of the system and are ideally suited to analysis using geometric ideas.

It is convenient to introduce new phase variables $\theta_{n}=\phi_{n}+\Omega t$ so that (24) may be re-written as

$$
\dot{\phi}_{n}=\epsilon \sum_{m} W_{n m} R\left(\phi_{n}+\Omega t\right) X(t)
$$

In the weak coupling regime each oscillator approximately fires at its natural frequency $\Omega$. However, this relatively fast oscillation is slowly modulated by the $O(\epsilon)$ drift in the phases $\phi_{n}(t)$. We can expect to describe the output of the network in a periodic state with the ansatz $T_{n}^{j}=\left(j+\theta_{T}-\phi_{n}\right) T$ for some integer $j$ and period $T$ ( $\epsilon$-close to that of the natural period of the uncoupled oscillator). The right-hand side of equation (26) then becomes a $T$-periodic function of $t$ and we can apply the method of averaging [14]: there exists a change of variables, $\phi_{n} \rightarrow \phi_{n}+\epsilon f\left(\phi_{n}, t, \epsilon\right)$ that maps solutions of (26) to those of

$$
\frac{\mathrm{d} \phi_{n}}{\mathrm{~d} t}=\epsilon \sum_{m} W_{n m} H\left(\phi_{m}-\phi_{n}\right)+O\left(\epsilon^{2}\right),
$$

where $H(\phi)$ is the phase interaction function

$$
H(\phi)=\frac{1}{T} \int_{0}^{T} R\left(t / T+\theta_{T}\right) P(t+\phi T) \mathrm{d} t
$$

and

$$
P(t)=\sum_{j} \eta(t-j T), \quad t \in[0, T) .
$$

The function $P(t)$ is periodically extended such that $P(t+T)=P(t)$. In neural models the effective phase interaction may be regarded as a convolution (over one period of oscillation) of the synaptic current and the so-called phase response curve (PRC) of a single neuron. The PRC can be found experimentally by perturbing a neuron with a brief depolarizing stimulus at different points on its limit cycle and measuring the resulting phase-shift. For certain types of neuron such a stimulus always advances the onset of the next spike, that is, the PRC is always positive, whereas for others the stimulus may also delay the next spike. Differences in the nature of a neuron's PRC are known to have a major effect on phase dynamics at the network level [17]. 
The method of averaging is only expected to yield sensible results in the limit $\epsilon \ll \mu \rightarrow 0$ (see [37] for a discussion). In general the function $f(\phi, t, \epsilon)$ is not small when $t \rightarrow \infty$. However, for $\epsilon \ll 1$, the dynamics of (26) are $\epsilon$-close to those of $(27)$ for times of $O\left(\epsilon^{-1}\right)$. Moreover, hyperbolic periodic orbits of (27) correspond to hyperbolic periodic orbits of (26). Consider, in particular, phase-locked solutions of (27) for which $\phi_{n}(t)=\psi_{n}+\widetilde{\Omega} t$, where $\psi_{n}$ is a constant phase and $\widetilde{\Omega}$ is an $O(\epsilon)$ contribution to the effective frequency of the oscillators. Substituting into (27) and working to $O(\epsilon)$ leads to the fixed point equations

$$
\widetilde{\Omega}=\epsilon \sum_{m} W_{n m} H\left(\psi_{m}-\psi_{n}\right)
$$

The neural firing times in the phase-locked state are given by $T_{n}^{j}=\left(j+\theta_{T}-\psi_{n}\right) T$, with $T^{-1}=\Omega+\widetilde{\Omega}$. For intermediate levels of coupling, where the method of averaging breaks down, one must deal directly with (26) in which the interaction between neurons involves the direct product of the PRC with the post-synaptic current, $X_{m}(t)=P\left(t+\phi_{m}-\theta_{T}\right)$, such that the phases no longer occur as differences. This generally makes the analysis far more difficult and will not be pursued here.

The (linear) stability of solutions is determined by setting $\phi_{n}(t)=\psi_{n}+\widetilde{\Omega} t+\varphi_{n}(t)$ to obtain

$$
\frac{\mathrm{d} \varphi_{n}}{\mathrm{~d} t}=\sum_{m} \mathcal{H}_{n m}(\Psi)\left[\varphi_{m}-\varphi_{n}\right]
$$

where $\mathcal{H}_{n m}(\Psi)=\epsilon W_{n m} H^{\prime}\left(\psi_{m}-\psi_{n}\right)$. Hence stability is determined by the eigenvalues of the Jacobian matrix $\widehat{\mathcal{H}}_{n m}(\Psi)=\mathcal{H}_{n m}(\Psi)-\delta_{n m} \sum_{k} \mathcal{H}_{n k}(\Psi)$.

We finish the construction of the phase interaction function by writing the response function (for $\theta \neq 0, \theta_{T}$ ) in the form

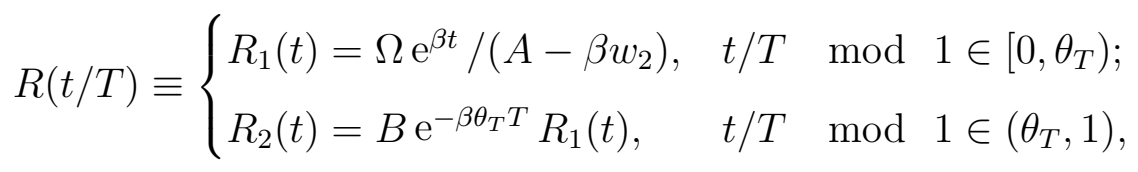

where $B=\left(A-\beta w_{2}\right) /\left(A+1-\beta w_{1}\right)$. Then, using the periodicity properties of $P(t)$ and 
$R(t)$ we may write

$$
H(\phi)-\widehat{H}(\phi)=\left\{\begin{array}{l}
B F(\phi ; 0,1-\phi, 0) \\
+B F\left(\phi ; 1-\phi, 1-\theta_{T},-1\right) \\
+\mathrm{e}^{\beta\left(\theta_{T}-1\right) T} F\left(\phi ; 1-\theta_{T}, 1,-1\right), \quad \phi>\theta_{T} ; \\
B F\left(\phi ; 0,1-\theta_{T}, 0\right) \\
+\mathrm{e}^{\beta\left(\theta_{T}-1\right) T} F\left(\phi ; 1-\theta_{T}, 1-\phi, 0\right) \\
+\mathrm{e}^{\beta\left(\theta_{T}-1\right) T} F(\phi ; 1-\phi, 1 ;-1), \quad \phi<\theta_{T} .
\end{array}\right.
$$

where

$$
F(\phi ; a, b, c)=\frac{1}{T} \int_{a}^{b} R_{1}(t) P(t+\phi T+c T) \mathrm{d} t
$$

and

$$
\widehat{H}(\phi)=\Omega^{2}\left[\kappa(0) P\left(\left(\phi-\theta_{T}\right) T\right)+\kappa\left(\theta_{T}\right) P(\phi T)\right] .
$$

Note that $\widehat{H}(\phi)$ is discontinuous, with discontinuities at $\phi=0$ and $\phi=\theta_{T}$, since $P(0) \neq$ $P(T)$. For a general discussion of discontinuous phase interaction functions in relaxation oscillator systems see the recent paper by Izhikevich [37].

\section{Phase locking in a pair of mutually coupled binary relaxation oscillators}

In this section we illustrate the theory of weakly connected McKean oscillators for a pair with reciprocal connections and an alpha function synaptic kernel. An alpha function is a common way of representing a synaptic response when the rise and fall time of the synapse is comparable. For this model of the synaptic transmission process we set $\eta(t)=$ $\alpha^{2} t \mathrm{e}^{-\alpha t} \Theta(t)$. The calculation of the interaction function for this case is straightforward since we may sum the infinite series (29) to obtain

$$
P(t)=\frac{\alpha^{2} \mathrm{e}^{-\alpha t}}{\left(1-\mathrm{e}^{-\alpha T}\right)}\left[t+\frac{T \mathrm{e}^{-\alpha T}}{1-\mathrm{e}^{-\alpha T}}\right], \quad t \in[0, T) .
$$

The calculation of (34) is also straightforward and yields

$$
F(\phi ; a, b, c)=\frac{\alpha^{2} \mathrm{e}^{-\alpha(\phi+c) T}}{T^{2}\left(1-\mathrm{e}^{-\alpha T}\right)(\beta-\alpha)\left(A-\beta w_{2}\right)} M(\phi ; a T, b T, c)
$$



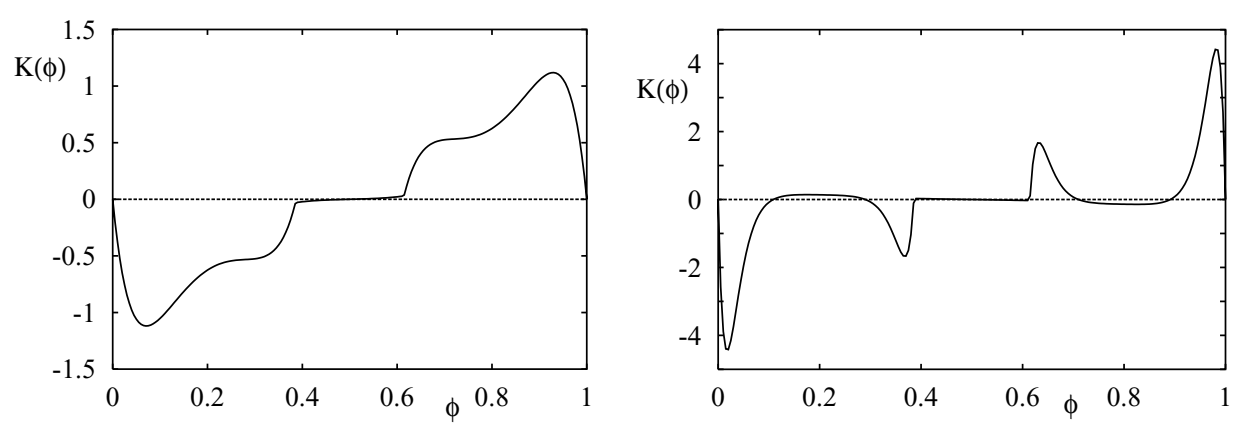

Fig. 3. The function $K(\phi)=H(1-\phi)-H(\phi)$ for two synaptically coupled binary oscillators with alpha function kernels. $v_{0}=w_{0}=0, \gamma=0.5, I=0.5$. On the left $\alpha=5$ and on the right $\alpha=20$.

with

$$
\begin{aligned}
M(\phi ; a, b, c) & =\left[(\phi+c) T+\frac{T \mathrm{e}^{-\alpha T}}{\left(1-\mathrm{e}^{-\alpha T}\right)}-\frac{1}{(\beta-\alpha)}\right]\left(\mathrm{e}^{b(\beta-\alpha)}-\mathrm{e}^{a(\beta-\alpha)}\right) \\
& +\left(b \mathrm{e}^{b(\beta-\alpha)}-a \mathrm{e}^{a(\beta-\alpha)}\right) .
\end{aligned}
$$

For the study of phase-locking it is natural to follow the evolution of the phase difference $\phi=\phi_{1}-\phi_{2}$. Using (27) we have that

$$
\dot{\phi}=\epsilon K(\phi) \equiv \epsilon[H(1-\phi)-H(\phi)]
$$

By symmetry the synchronous solution $\phi=0$ and the anti-synchronous solution $\phi=1 / 2$ are guaranteed to exist for all system parameter values. Other phase-locked solutions, which we term asynchronous, will satisfy $K(\phi)=0$ with $1-\phi$ also a solution if $\phi$ is a solution. Solutions are linearly stable if $\epsilon K^{\prime}(\phi)<0$. Some numerical plots of the function $K(\phi)$ are shown in figure 3. For large values of $\alpha$ one finds three other types of phaselocked solution apart from the synchronous and anti-synchronous solution. However with decreasing $\alpha$ these solutions can cease to exist. By examining the derivative of $K(0)$ it is simple to determine that the synchronous solution is stable for all $\alpha$ when $\epsilon>0$ (excitatory coupling) and unstable when $\epsilon<0$ (inhibitory coupling). The full bifurcation diagram for the system, as a function of the inverse rise time $\alpha$, is presented in figures 4 and 5 . Here we present results for excitatory coupling and use solid lines to represent stable solutions and dashed lines for unstable. For inhibitory coupling the stability of solution branches is simply reversed. In common with phase oscillator studies of Hodgkin-Huxley neurons with mutual weak excitatory coupling through alpha functions [18] we find that for fast synapses the synchronous and anti-synchronous states can co-exist stably. The existence 


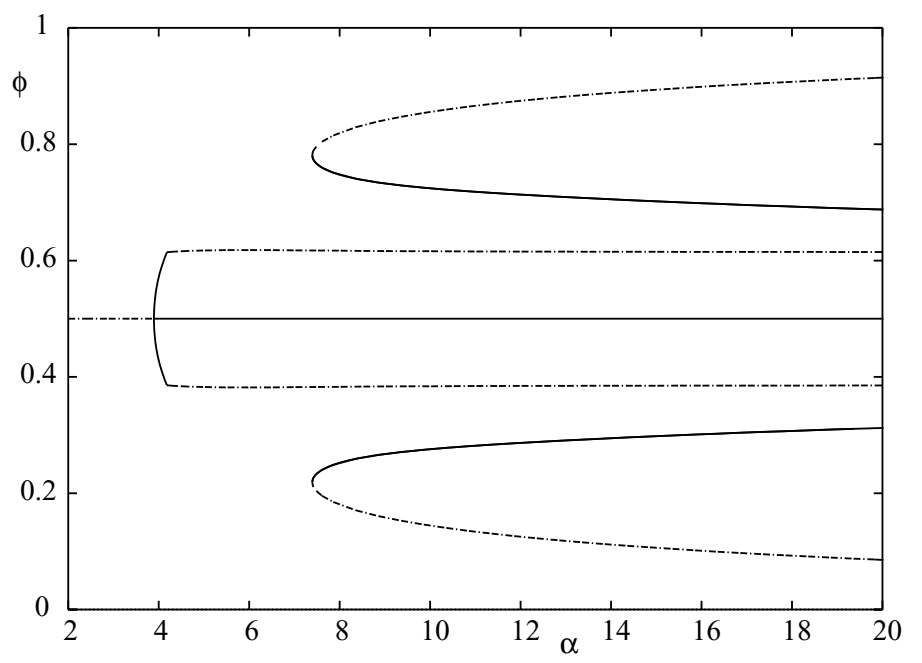

Fig. 4. Bifurcation diagram for two synaptically coupled binary oscillators with $\alpha>2$. $v_{0}=w_{0}=0, \gamma=0.5, a=0.25, I=0.5$. Solid (dashed) lines are stable (unstable).

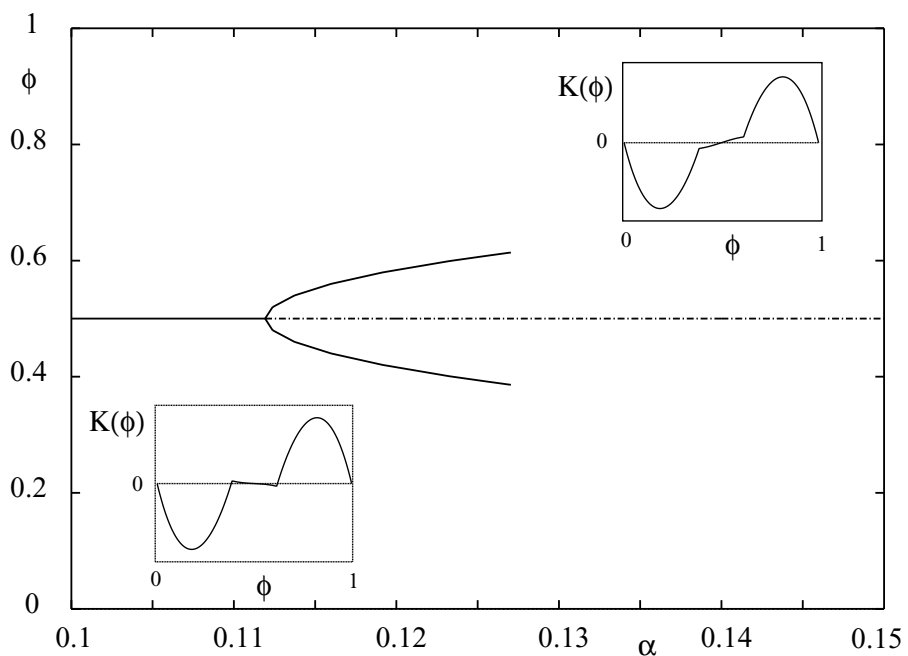

Fig. 5. Bifurcation diagram for two synaptically coupled binary oscillators for small $\alpha$. $v_{0}=w_{0}=0, \gamma=0.5, a=0.25, I=0.5$. Solid (dashed) lines are stable (unstable). The insets show the shape of the interaction function $K(\phi)$ away from the pitchfork bifurcation. Note that $K^{\prime}(0)$ does not change sign whilst $K^{\prime}(1 / 2)$ does. Hence, for excitatory coupling the synchronous solution is always stable and the anti-synchronous solution may be stable for very small $\alpha$. For larger $\alpha$ the anti-synchronous solution can destabilize via a pitchfork bifurcation. The stability of solution branches is reversed for inhibitory coupling. 


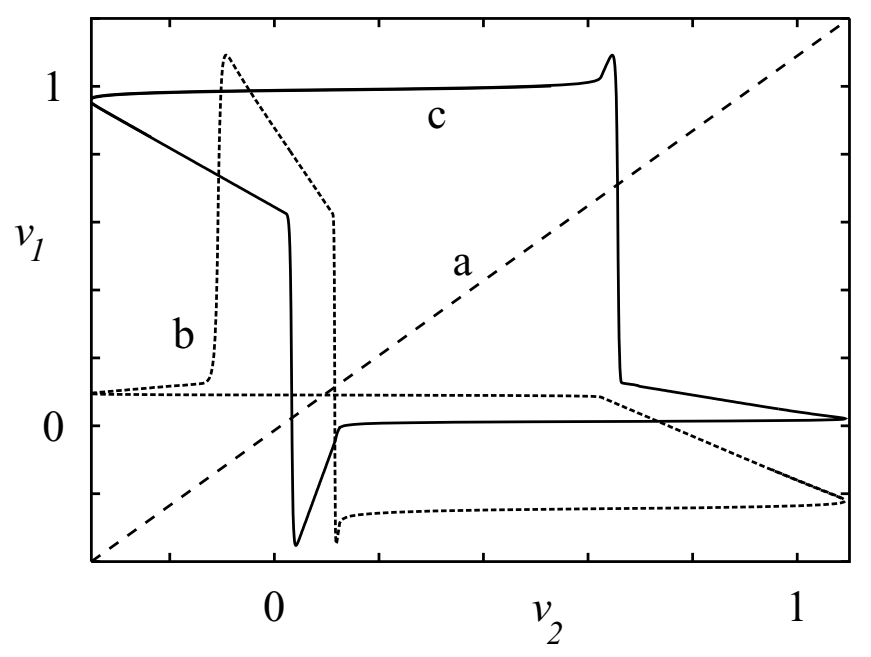

Fig. 6. Numerical simulation of a pair of synaptically coupled McKean neurons showing co-existence of the synchronous (a), anti-synchronous (b) and one asynchronous state (c) for large $\alpha(\alpha=20)$. Other parameters $v_{0}=w_{0}=0, a=0.25, \gamma=0.5, I=0.5, \mu=0.01$ and $\epsilon=0.001$.

of an unstable asynchronous solution that can connect to the anti-synchronous state with decreasing $\alpha$ (at around $\alpha=4$ ) is also a phenomenon seen in the weakly coupled HodgkinHuxley pair. For slow synapses the anti-synchronous solution is unstable (see figure 4 with $2<\alpha<4)$.

For extremely slow synapses (see figure 5) the anti-synchronous solution can change stability type in a pitchfork bifurcation. Once again just this type of bifurcation has been observed with Hodgkin-Huxley dynamics. One difference to the simulations presented in [18] is that the asynchronous states, seen for very slow synapses, do not reconnect to the synchronous solution. Rather the underlying discontinuous nature of the phase interaction function means that they can cease to exist as $\alpha$ is varied, preventing reconnection. This subtle difference is associated with the fast relaxation limit $\mu \rightarrow 0$, which is not expected to capture all the essential features of the usual Hodgkin-Huxley dynamics with standard parameter values. The appearance of a pair of stable/unstable phase-locked solutions at high $\alpha$ is also expected to be another artifact of the fast relaxation assumption, since these states were not seen in the numerics presented in [18]. A direct numerical example of this particular type of phase-locked state, in conjunction with a synchronous and anti-synchronous orbit is shown in figure 6 .

For numerical simulation, and considering a synaptic kernel described with an alpha 
function, it is convenient to rewrite (25) in the form

$$
\begin{aligned}
& \frac{1}{\alpha} \frac{\mathrm{d} X_{m}}{\mathrm{~d} t}=-X_{m}+Y_{m} \\
& \frac{1}{\alpha} \frac{\mathrm{d} Y_{m}}{\mathrm{~d} t}=-Y_{m}+\sum_{j} \delta\left(t-T_{j}^{m}\right)
\end{aligned}
$$

where we have made use of the fact that $\eta(0)=0$. A practical way in which to simulate this second order synapse is therefore to evolve the system of equations (40) and (41) until a firing event is reached and then to discontinuosly adjust $Y_{m}$ according to $Y_{m} \rightarrow Y_{m}+\alpha$. Throughout we have assumed that the input $\epsilon X_{n}(t)$ is insufficient to switch the dynamics from the branch $S=0$ to $S=1$. The possibility of a transition from the branch $S=0$ to $S=1$ at other phase than $\theta=\theta_{T}$ will be considered next. Importantly, new phase-locked phenomenona, most notably the existence a continuum of stable asynchronous states, becomes possible for strong coupling in the limit $\alpha \rightarrow \infty$ and $\mu=0$.

\section{$5 \quad$ Strong pulsatile coupling}

In this section we show how the binary model can be interpreted as a type of IF oscillator with a state-dependent threshold and recovery variable. In this framework it is possible to treat the case of fast pulsatile synaptic interactions (where $\eta(t) \rightarrow \delta(t)$ ) with a return-map analysis $[40,45,46,47,48]$ The state dependent threshold function of the binary model is identified as the middle part of the $\dot{v}=0$ nullcline described by the linear equation $v=v_{c}(w)$ (see figure 1). Initial data with $v<v_{c}(w)$ will be attracted to the $S=0$ branch, whilst if $v>v_{c}(w)$ it is attracted to the $S=1$ branch. Hence, if a binary neuron evolves freely on $S=0$ until $v$ is perturbed by a pulse of strength $\kappa$ then it will make a transition to the other branch only if $v+\kappa>v_{c}(w)$. If it fails to make a transition or if it is stimulated whilst on the branch $S=1$ it will instantaneously relax back to its original branch. Hence, even though stimulated it would appear to behave as if it were evolving freely (at least in the singular limit $\mu=0$ ). Note that is very different from the case of weak coupling where the assumption was made that jumps from $S=0$ to $S=1$ could only occur when $w=w_{1}$. We may keep track of which branch the system is on by writing

$$
S_{n}^{+}=\Theta\left(S_{n}-h\left(w_{n}, \kappa_{n}\right)\right),
$$


where

$$
\kappa_{n}(t)=\sum_{m} W_{n m} \sum_{j} \delta\left(t-T_{m}^{j}\right), \quad h(w, \kappa)=2 w+a-\kappa-2\left(A+v_{0}\right) .
$$

The parameters $W_{n m}$ are to be interpreted as the size of the kicks communicated by oscillator $m$ to oscillator $n$. The delta function may be regarded as the large $\alpha$ limit of the alpha function of the previous section. Transitions from $S=0$ to $S=1$ will occur whenever $w=w_{1}$ or if the system is kicked with a strength $\kappa$ whilst in the state $w_{n}$ and $w_{n}<w_{D}$, where $w_{D}$ is the solution to $h\left(w_{D}, \kappa\right)=0$, ie $w_{D}=A+v_{0}+(\kappa-\alpha) / 2$. The dynamics of a network of pulse-coupled binary neurons may now be written in the simple form

$$
\dot{w}_{n}=-\beta w_{n}+A+S_{n},
$$

with $S_{n}$ determined by (42).

For further analysis it is convenient to describe the system in terms of the phase variables introduced in the previous section. Note that on the branch $S=0$ we may write

$$
\theta=f(w)=\frac{1}{\beta T} \ln \left[\frac{-\beta w_{2}+A}{-\beta w+A}\right]
$$

This allows us to define an impact phase $\theta_{D}=f\left(w_{D}\right)$ (for a kick of strength $\kappa$ ) such that the phase just after a neuron receives a pulse of strength $\kappa$ is given by

$$
\theta^{+}=F^{+}(\theta)= \begin{cases}\theta, & \theta<\theta_{D} \\ F(\theta), & \theta_{D} \leq \theta<\theta_{T} \\ \theta, & \theta \geq \theta_{T}\end{cases}
$$

We take $\kappa \leq 1$ so that $0<\theta_{D}<\theta_{T} . F(\theta)$ is simply the new phase after jumping from the branch $S=0$ to $S=1$ :

$$
F(\theta)=1-\frac{1}{T} \int_{g(\theta)}^{w_{2}} \frac{\mathrm{d} w}{G(w ; 1)}=\frac{1}{\beta T} \ln \left[\frac{1-\nu}{1-\nu \mathrm{e}^{-\beta \theta T}}\right],
$$

where $g(\theta)=f^{-1}(\theta)$ and $\nu=\beta w_{2}-A$.

We shall now illustrate how this system may be analyzed with a return-map approach by considering the evolution of a pair of excitatory pulse-coupled binary oscillators. We introduce two neurons with phases $\theta_{1}$ and $\theta_{2}$ respectively and take initial data such that $\left(\theta_{1}(0), \theta_{2}(0)\right)=\left(\phi, \theta_{T}\right)$. Just after the firing of neuron 2 we have that $\left(\theta_{1}, \theta_{2}\right)=$ 

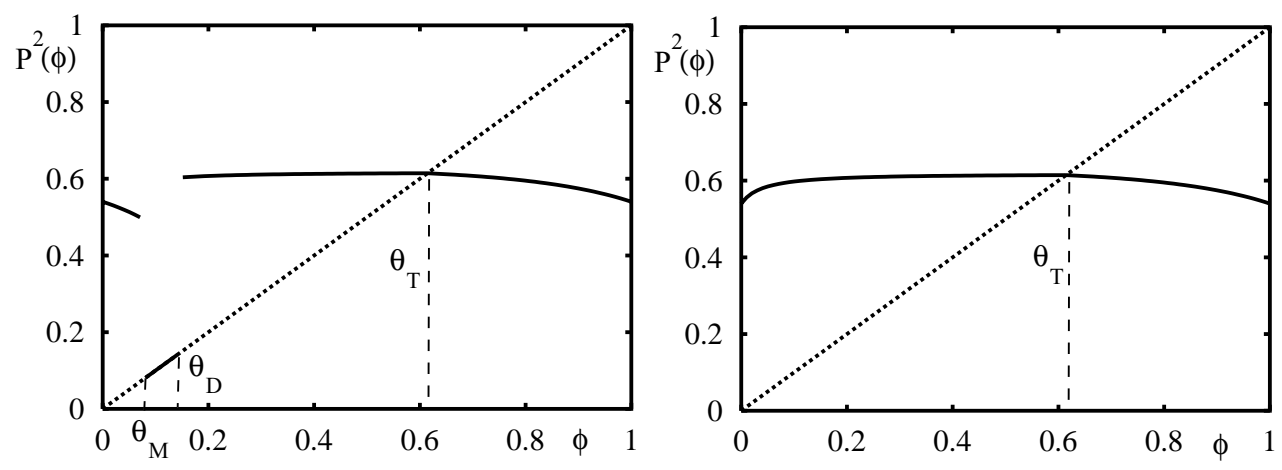

Fig. 7. Return-map for a pair of binary neurons with mutual excitation. $v_{0}=w_{0}=0, \gamma=0.5$, $a=0.25, I=0.5$. On the left $\kappa=0.5$ and on the right $\kappa=1$.

$\left(F^{+}(\phi), \theta_{T}\right)$. If we evolve the system until neuron 1 fires then $\left(\theta_{1}, \theta_{2}\right)=\left(\theta_{T}, P(\phi)\right)$, where

$$
P(\phi)=2 \theta_{T}-F^{+}(\phi)+1 \bmod 1 .
$$

Thus after one firing, the system has moved from an initial state $\left(\phi, \theta_{T}\right)$ to a current state $\left(\theta_{T}, P(\phi)\right)$. Hence the system is in essentially the same state as when it started, but with $\phi$ replaced by $P(\phi)$ and the oscillators interchanged. The so-called return-map [40] for the system is obtained by following the system through one more firing event and is simply $P^{2}(\phi)$. Using the observation that $F^{+}\left(\theta_{T}\right)=\theta_{T}$ it is a simple matter to establish that one fixed point of the return-map, $P^{2}(\phi)=\phi$, is the synchronous solution $\phi=\theta_{T}$. The graph of the return-map is shown in figure 7 and illustrates that as well as the synchronous solution there are asynchronous solutions with $\phi \in\left(\theta_{M}, \theta_{D}\right), \theta_{M} \in\left(0, \theta_{D}\right)$. Interestingly, a continuum of states is also observed for pulse-coupled IF neurons with the inclusion of a refractory period [49]. The reason why there is a continuum of asynchronous solutions can be traced to the fact that a neuron with phase $\phi \in\left(0, \theta_{D}\right)$ does not feel the effect of a firing event (at least for instantaneous relaxation where $\mu=0$ ). If neuron one has some phase $\phi \in\left(\theta_{M}, \theta_{D}\right)$ when neuron two fires, then neuron two will also be immune to the firing of neuron one only if $\theta_{T}+\left(\theta_{T}-\phi\right) \bmod 1<\theta_{D}$. Hence, one may easily calculate that $\theta_{M}=2 \theta_{T}-1-\theta_{D}$. The system as a whole then behaves as if there were no coupling and the relative phase between oscillators is completely specified by initial data and will remain fixed for all time. In the limit $\theta_{D} \rightarrow 0$ (or equivalently $\kappa \rightarrow 1$ ) the only remaining fixed point of the return-map is the synchronous solution. In common with the results for FTM coupling, synchronization is robust and can be extremely rapid. The FTM theory applies to relaxation oscillators with cubic fast nullclines, fast relaxation and synaptic kernels in the form of Heaviside step functions. In common with the analysis of 

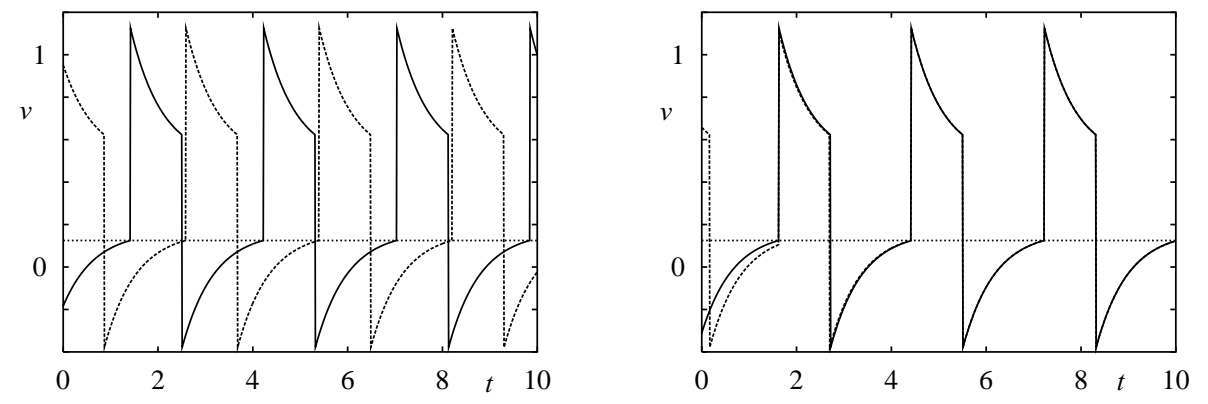

Fig. 8. Evolution to an asynchronous solution $(\kappa=0.5)$ and a synchronous solution $(\kappa=1)$ $a=0.25, I=0.5, \gamma=0.5, v_{0}=0, w_{0}=0$.

this section there is no restriction on the strength of coupling. However, unlike the FTM theory we do not seem to have to invoke the compression hypothesis in which the rate of change of the slow variable must decrease through a jump between slow manifolds. An example of the evolution into a synchronous and asynchronous state for a pair of pulse-coupled binary oscillators is shown in figure 8 .

Note that if $\kappa>1$ then the firing of one neuron will guarantee the firing of its partner. Even though they may not have the same phase the two oscillators are synchronized in the sense that they always fire at the same time. Moreover, they will synchronize, in this weaker sense, at the first firing event.

\section{Discussion}

The McKean model is a low-dimensional and uncomplicated neural relaxation oscillator, originally introduced as a minimal model of excitable neural tissue relevant for describing action potential propagation in models of spatially extended axons [35]. We have now shown that it is possible to explicitly calculate the PRC of a point McKean oscillator using simple geometric notions. Unlike oscillators of non-relaxation type, the PRC is seen to be discontinuous, as predicted by Izhikevich [37]. This discontinuous PRC forms the basis of a description of networks of synaptically interacting oscillatory McKean neurons. Under the assumption of fast relaxation and weak coupling it has been possible to analyze the way in which synaptic and recovery time scales interact to generate phase-locked states. An application of the general theory to a pair with mutual coupling through alpha functions is found to be consistent with earlier numerical studies of Hodgkin-Huxley networks. 
This lends further support to the usefulness of the fast relaxation limit of the McKean oscillator in providing a sound starting point for the mathematical analysis of networks of biologically motivated spiking neural oscillators. The application of this work to cover specific neuronal architectures and synaptic kernels (such as those described in [44]) is relevant to the study of phase-locked solutions of neural systems, solutions that arise through instabilities, and indeed phase waves of the type discussed by Crook et al. [50]. A more challenging mathematical problem is the development of a theory of strongly coupled relaxation oscillators (perhaps without recourse to the fast relaxation assumption). One step in this direction has also been presented in this paper, and shows that the synchronous solution can co-exist with a continuum of asynchronous states. Unfortunately the study of pulse-coupled binary oscillators, via a return-map analysis, would not seem to lend itself to a study of non-instantaneous synapses. However, recent techniques developed for the numerical study of synaptically interacting Hodgkin-Huxley neurons [51], when combined with the PRC of the McKean model, may allow such a programme to be developed. These and related issues are topics of current investigation.

\section{Acknowledgements}

The author would like to acknowledge financial support from the Nuffield Foundation and to thank Bard Ermentrout for making available the dynamical systems software tool XPP.

\section{References}

[1] M Steriade, E G Jones, and R R Llinás. Thalamic Oscillations and Signalling. Wiley, 1990.

[2] M A Whittington, R D Traub, and J G R Jefferys. Synchronized oscillations in interneuron networks driven by metabotropic glutamate receptor activation. Nature, 373:612-615, 1995.

[3] T G Brown. On the nature of the fundamental activity of the nervous centres; together with an analysis of the conditioning of rhythmic activity in progression, and a theory of the evolution of function in the nervous system. Journal of Physiology, 48:18-46, 1914.

[4] C M Gray, P König, A K Engel, and W Singer. Oscillatory responses in cat visual cor- 
tex exhibit intercolumnar synchronization which reflects global stimulus properties. Nature, 338:334-337, 1989.

[5] D Golomb, X J Wang, and J Rinzel. Synchronization properties of spindle oscillations in a thalamic reticular nucleus model. Journal of Neurophysiology, 72:1109-1126, 1994.

[6] W Lytton and T J Sejnowski. Simulations of cortical pyramidal neurons synchronized by inhibitory neurons. Journal of Neurophysiology, 66:1059-1097, 1991.

[7] A Destexhe, D Contreras, T J Sejnowski, and M Steriade. A model of spindle rhythmicity in the isolated thalamic reticular nucleus. Journal of Neurophysiology, 72:803818, 1994.

[8] P Bush and T J Sejnowski. Inhibition synchronizes sparsely connected cortical neurons within and between columns of realistic network models. Journal of Computational Neuroscience, 3:91-110, 1996.

[9] J G R Jefferys, R D Traub, and M A Whittington. Neuronal networks for induced '40 Hz' rhythms. Trends in Neuroscience, 19:202-207, 1996.

[10] X J Wang and G Buzsaki. Gamma oscillation by synaptic inhibition in a hippocampal interneuronal network. Journal of Neuroscience, 16:6402-6413, 1996.

[11] R D Traub, J G R Jefferys, and M A Whittington. Fast Oscillations in Cortical Circuits. MIT Press, 1999.

[12] A L Hodgkin and A F Huxley. A quantitative description of membrane current and its application to conduction and excitation in nerve tissue. Journal of Physiology (London), 116:449-472, 1952.

[13] N Fenichel. Persistence and smoothness of invariant manifolds for flows. Indiana University Mathematics Journal, 21:193-225, 1971.

[14] J Guckenheimer and P Holmes. Nonlinear Oscillations, Dynamical Systems, and Bifurcations of Vector Fields. Springer, 1983.

[15] Y Kuramoto. Chemical Oscillations, Waves and Turbulence. Springer-Verlag, 1984.

[16] G B Ermentrout and N Kopell. Frequency plateaus in a chain of weakly coupled oscillators. SIAM Journal on Applied Mathematics, 15:215-237, 1984.

[17] D Hansel, G Mato, and C Meunier. Synchrony in excitatory neural networks. Neural Computation, 7:307-337, 1995.

[18] C van Vreeswijk, L F Abbott, and G B Ermentrout. When inhibition not excitation synchronizes neural firing. Journal of Computational Neuroscience, 1:313-321, 1994.

[19] C van Vreeswijk. Partial synchronization in populations of pulse-coupled oscillators. 
Physical Review E, 54:5522-5537, 1996.

[20] W Gerstner, J L van Hemmen, and JD Cowan. What matters in neuronal locking. Neural Computation, 94:1653-1676, 1996.

[21] S Coombes and G J Lord. Desynchronization of pulse-coupled integrate-and-fire neurons. Physical Review E, 55(3):R2104-R2107, 1997.

[22] S Coombes and G J Lord. Intrinsic modulation of pulse-coupled integrate-and-fire neurons. Physical Review E, 56(5):5809-5818, 1997.

[23] C C Chow. Phase-locking in weakly heterogeneous neuronal networks. Physica D, 118(3-4):343-370, 1998.

[24] J A White, C C Chow, J Ritt, C Soto-Trevino, and N Kopell. Synchronization and oscillatory dynamics in heterogeneous, mutually inhibited neurons. Journal of Computational Neuroscience, 5:5-16, 1998.

[25] P C Bressloff and S Coombes. Dynamics of strongly coupled spiking neurons. Neural Computation, 12:91-129, 2000.

[26] D Terman and D Wang. Global competition and local cooperation in a network of neural oscillators. Physica D, 81:148-176, 1995.

[27] D Somers and N Kopell. Waves and synchrony in networks of oscillators of relaxation and non-relaxation type. Physica D, 89:169-183, 1995.

[28] D Terman, N Kopell, and A Bose. Dynamics of two mutually coupled slow inhibitory neurons. Physica D, 117:241-275, 1998.

[29] T LoFaro and N Kopell. Timing regulation in a network reduced from voltage-gated equations to a one-dimensional map. Journal of Mathematical Biology, 38:479-533, 1999.

[30] J Rubin and D Terman. Geometric analysis of population rhythms in synaptically coupled neuronal networks. Neural Computation, 12:597-645, 2000.

[31] J Rubin and D Terman. Analysis of clustered firing patterns in synaptically coupled networks of oscillators. Journal of Mathematical Biology, (to appear), 2000.

[32] A Bose, N Kopell, and D Terman. Almost-synchronous solutions for mutually coupled excitatory neurons. Physica D, 140:69-94, 2000.

[33] J Cronin and R E O'Malley Jr, editors. Analysing Multiscale Phenomena Using Singular Perturbation Methods, volume 56 of Proceedings of Symposia in Applied Mathematics. American Mathematical Society, 1999.

[34] J Rubin and D Terman. Geometric singular perturbation analysis of neuronal dynamics. In B Fiedler, G Ioos, and N Kopell, editors, Handbook of Dynamical Systems, 
volume 3. to appear, 2000.

[35] H P McKean. Nagumo's equation. Advances in Mathematics, 4:209-223, 1970.

[36] L F Abbott. A network of oscillators. Journal of Physics A, 23:3835-3859, 1990.

[37] E M Izhikevich. Phase equations for relaxation oscillators. SIAM Journal on Applied Mathematics, 60:1789-1805, 2000.

[38] D Somers and N Kopell. Rapid synchronization through fast threshold modulation. Biological Cybernetics, 68:393-407, 1993.

[39] N Kopell and D Somers. Anti-phase solutions in relaxation oscillators coupled through excitatory interactions. Journal of Mathematical Biology, 33:261-280, 1995.

[40] R E Mirollo and S H Strogatz. Synchronization of pulse-coupled biological oscillators. SIAM Journal on Applied Mathematics, 50:1645-1662, 1990.

[41] S Coombes and A H Osbaldestin. Period adding bifurcations and chaos in a periodically stimulated excitable neural relaxation oscillator. Physical Review E, 62:40574066, 2000.

[42] G B Ermentrout and N Kopell. Oscillator death in systems of coupled neural oscillators. SIAM Journal on Applied Mathematics, 50:125-146, 1990.

[43] G B Ermentrout and N Kopell. Multiple pulse interactions and averaging in systems of coupled neural oscillators. Journal of Mathematical Biology, 29:195-217, 1991.

[44] P C Bressloff and S Coombes. Symmetry and phase-locking in a ring of pulse-coupled oscillators with distributed delays. Physica D, 126:99-122, 1999.

[45] S Bottani. Pulse-coupled relaxation oscillators: from biological synchronization to self-organized criticality. Physical Review Letters, 74:4189-4192, 1995.

[46] U Ernst, K Pawelzik, and T Geisel. Synchronization induced by temporal delays in pulse-coupled oscillators. Physical Review Letters, 74:1570-1573, 1995.

[47] R Mathar and J Mattfeldt. Pulse-coupled decentral synchronization. SIAM Journal on Applied Mathematics, 56:1094-1106, 1996.

[48] U Ernst, K Pawelzik, and T Geisel. Delay-induced multistable synchronization of biological oscillators. Physical Review E, 57:2150-2162, 1998.

[49] V Kirk and E Stone. Effect of a refractory period on the entrainment of pulse-coupled integrate-and-fire oscillators. Physic Letters A, 232:70-76, 1997.

[50] S M Crook, G B Ermentrout, M C Vanier, and J M Bower. The role of axonal delay in the synchronization of networks of coupled cortical oscillators. Journal of Computational Neuroscience, 4:161-172, 1997.

[51] T Yoshinaga, Y Sano, and H Kawakami. A method to calculate bifurcations in 
synaptically coupled Hodgkin-Huxley equations. International Journal of Bifurcation and Chaos, 9:1451-1458, 1999. 